\title{
Identification of a Time-varying Beam Using Hilbert Vibration Decomposition
}

\author{
M. Bertha, J.C. Golinval \\ University of Liège, Liège, Belgium \\ Department of Aerospace and Mechanical Engineering \\ mathieu.bertha@ulg.ac.be, jc.golinval@ulg.ac.be
}

\begin{abstract}
The present work is concerned by modal identification of time-varying systems. For this purpose, a method based on instantaneous frequency identification and synchronous demodulation is used to extract modal components from recorded signals. The proposed method of iterated sifting process is based on the Hilbert Vibration Decomposition (HVD) technique which is used to extract the instantaneous dominant vibrating component at each iteration. A source separation preprocessing step is introduced to treat multiple degree-of-freedom systems in an optimal way. Sources are used as reference signals to get a single instantaneous frequency of each mode for the demodulation on all the channels. The algorithm is presented and is applied to numerical simulation of a randomly excited time-varying structure for illustration purpose. The investigated structure is made up of a beam on which a non-negligible mass is traveling. The variable location of the mass results in changes in modal parameters.
\end{abstract}

Keywords: Modal identification, time-varying systems, instantaneous modal parameters, Hilbert transform, signal decomposition.

\section{INTRODUCTION}

During the last decade, a lot of new processing techniques appeared in the field of modal identification of time-varying systems. The Hilbert-Huang transform (HHT) introduced by Huang et al. in ${ }^{[1]}$ is a powerful and highly adaptive technique able to deal with non-stationary signals. First, the use of the Empirical Mode Decomposition (EMD) method results in a set of so-called Intrinsic Mode Functions (IMFs) which are ideally mono-component signals. Then the instantaneous frequency and instantaneous amplitude of each IMF are obtained by use of the Hilbert transform.

More recently, the Hilbert Vibration Method (HVD) proposed by Feldman ${ }^{[2]}$ was introduced with the ambition of being more accurate than the EMD method. In the following, the HVD method will serve as a basis for the developments reported in the present paper.

The paper is organized as follows. First the main ideas of the EMD and HVD methods are recalled and their practical limitations are highlighted. Then a description of the proposed method is presented along with its application on synthetic responses of a randomly excited time-varying system. The system considered here consists in a beam simply supported at its both ends on which a non-negligible mass is moving. Finally, a conclusion and some words on future work close the paper. 


\section{THE HILBERT-HUANG TRANSFORM AS A TOOL TO COMPUTE INSTANTANEOUS PROPERTIES OF MULTI- COMPONENT SIGNALS}

The Hilbert-Huang transform works in two steps: 1) the signal is decomposed in IMFs using the EMD and 2) the instantaneous properties of the IMFs are extracted using the Hilbert transform.

\subsection{The empirical mode decomposition as sifting process}

The main idea of the HHT method is to sift the processed signal by successively removing slow oscillations from the signal using the EMD method. Practically, the EMD method works by first identifying all the minima and maxima in the signal. With the minima and maxima, the lower and upper envelopes of the signal are calculated by use of cubic-spline fitting, respectively. The mean of the lower and upper envelopes is a slowly oscillating function which is removed from the original signal. The process is then repeated until the remaining signal has the following two properties ${ }^{[1]}$ :

- the difference between the number of extrema and zero crossing is maximum 1 ,

- the local mean value of the lower and upper envelope of the signal is zero.

When these two conditions are fulfilled, the component is considered as an IMF and is extracted from the original signal. The process is iterated until it is not possible anymore to extract an IMF.

Once all useful IMFs are extracted, their instantaneous characteristics are calculated by use of the Hilbert transform.

\subsection{The Hilbert transform and the analytic signal for the extraction of instantaneous characteristics}

Let $x(t)$ be a real-valued signal. Its Hilbert transform $\mathcal{H}(x)$ is defined as the convolution product between the signal and $h(t)=\frac{1}{\pi t}:$

$$
y(t)=\mathcal{H}(x)=\frac{1}{\pi} \text { p.v. } \int_{-\infty}^{+\infty} \frac{x(\tau)}{t-\tau} \mathrm{d} \tau
$$

The Cauchy principal value of the integral has to be taken to solve this improper integral because of the singularity occurring for $\tau=t$.

A (non-exhaustive) list of properties of the Hilbert transform are given hereafter:

Inverse transform: $\mathcal{H}(\mathcal{H}(x))=-x$ which implies that $\mathcal{H}^{-1}=-\mathcal{H}$;

Differentiation: the $\left(\mathrm{k}^{\text {th }}-\right)$ derivative of the Hilbert transform is the Hilbert transform of the $\left(\mathrm{k}^{\text {th }}-\right)$ derivative $\frac{\mathrm{d}^{k}}{\mathrm{~d} t^{k}}[\mathcal{H}(x)]=\mathcal{H}\left(\frac{\mathrm{d}^{k} x}{\mathrm{~d} t^{k}}\right)$;

The Bedrosian's theorem ${ }^{[3]}$ : the Bedrosian's theorem states that if a signal $x(t)$ is a product of two components; one slowly and the other fast varying, then the Hilbert transform of $x(t)$ is equal to the slowly varying function times the Hilbert transform of the fast varying one. So, if $f(t)$ and $g(t)$ represent the slowly and fast varying functions and $x(t)=f(t) g(t)$, then $\mathcal{H}(x(t))=f(t) \mathcal{H}(g(t))$. The separation condition between slow and fast parts is that the Fourier spectra of $f$ and $g$ must not overlap.

By adding to the real-valued signal its Hilbert transform multiplied by the imaginary units, an analytic signal $z(t)$ is formed :

$$
z(t)=x(t)+i \mathcal{H}(x(t)) .
$$

In this form, the signal can now be seen as a rotating phasor in the complex plane characterized at each time instant by an amplitude $A(t)$ and a phase angle $\phi(t)$ :

$$
\begin{aligned}
z(t) & =x(t)+i \mathcal{H}(x(t)) \\
& =A(t) e^{i \phi(t)} .
\end{aligned}
$$


The instantaneous amplitude and phase are extracted directly from the analytic signal by the amplitude and argument calculations of $z$ :

$$
\begin{aligned}
A(t) & =|z(t)| ; \\
\phi(t) & =\angle z(t) ;
\end{aligned}
$$

and the instantaneous angular frequency of the signal is then obtained by time differentiation of the phase angle

$$
\omega(t)=\frac{\mathrm{d} \phi(t)}{\mathrm{d} t}
$$

In the case of a multi-component signal, the instantaneous amplitude and frequency obtained are those of the resultant signal (vector summation of each phasor). In that way, the amplitude of the signal is always lower or equal than the sum of the amplitudes of each component (triangle inequality) and the instantaneous frequency may be negative. This latter point occurs when the resultant phasor rotates backwards in the complex plane. Such a negative frequency has no physical meaning.

The HVD method presented in the next section uses the analytic form of the signal to split it into its mono-component parts.

\section{THE HILBERT VIBRATION DECOMPOSITION METHOD}

The Hilbert Vibration Decomposition method presented by M. Feldman ${ }^{[2]}$ is an iterative algorithm that exploits the analytic form to extract the dominant vibration component from a signal. The method is based on the fact that if we observe the phase (or frequency) of the analytic form of a multi-component signal, its global evolution is driven by the dominant mono-component (the one with the highest amplitude) of the signal. The other components appear as oscillations around the phase (frequency) of the dominant mode. It can be shown ${ }^{[2]}$ that the latter oscillation parts vanishes when integrated over the time. To isolate the dominant mode component present in the signal the phase (or frequency) is low-pass filtered. Finally, the component corresponding to the dominant mode is extracted from the signal using a synchronous demodulation based on the phase (or frequency) of the dominant mode. Once the dominant component is extracted, the whole process is repeated on the remaining components.

\section{DRAWBACKS OF THE HHT AND HVD METHODS}

For some multi-component signals the EMD and HVD methods may fail to sift properly all their constitutive mono-component signals. As recalled in Section 2.1 the EMD method extracts at each step the mono-component with the fastest oscillations. In Section 3 we saw that the HVD method extracts components from highest to lowest amplitudes. This implies that if the constitutive mono-component signals cross themselves in frequency or if the component with the highest frequency vanishes, the EMD method will not be able anymore to follow this component and will jump to the component having the new highest frequency. Similarly, if components cross themselves in amplitude the HVD method will always extract the component with the highest instantaneous amplitude.

To illustrate this problem, let us consider a simple two degree-of-freedom system which is made time-variant by introducing a time-dependent stiffness. The system is shown in Figure 1 and its properties are listed hereafter:

- $m_{1}=3 \mathrm{~kg}$;

- $m_{2}=1 \mathrm{~kg}$;

- $k_{1}=20000 \mathrm{~N} / \mathrm{m}$;

- $k_{2}=25000 \searrow 5000 \mathrm{~N} / \mathrm{m}$ (linear decrease in the time span);

- $c_{1}=3 \mathrm{Ns} / \mathrm{m}$.

The system is submitted to an impulse at DoF $x_{2}$ and the response of the whole system is simulated during 15 seconds. A standard Newmark integration scheme was used for the time integration in which the stiffness matrix is updated at each time step to take into account the dependence of $k_{2}$ with respect to the time. 

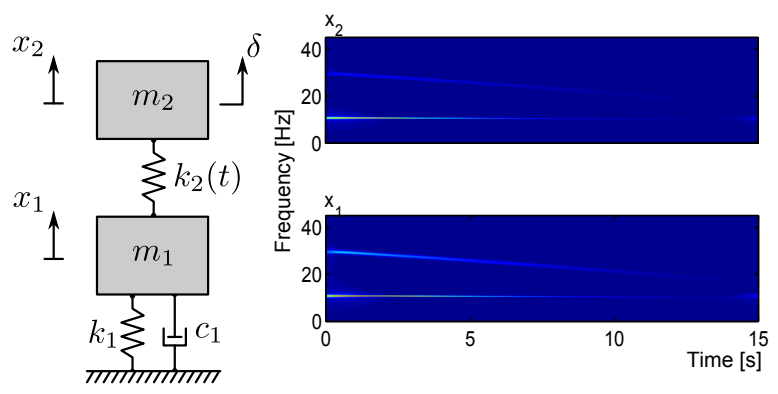

Figure 1: Two degree-of-freedom time-varying system with time-frequency plots of its time responses.

Applying the standard HVD method on the two channels separately leads to some undesirable behavior. Each time one intrinsic component becomes dominant in the signal, the method follows it and a mode switching phenomenon occurs during the extraction process. Moreover, as it can be seen in Figure 2, the mode switching does not occur at the same time on each channel, which makes the correction of these switches more difficult. When the frequency curve jumps from one mode to the other, the corresponding demodulated component also follows this jump.
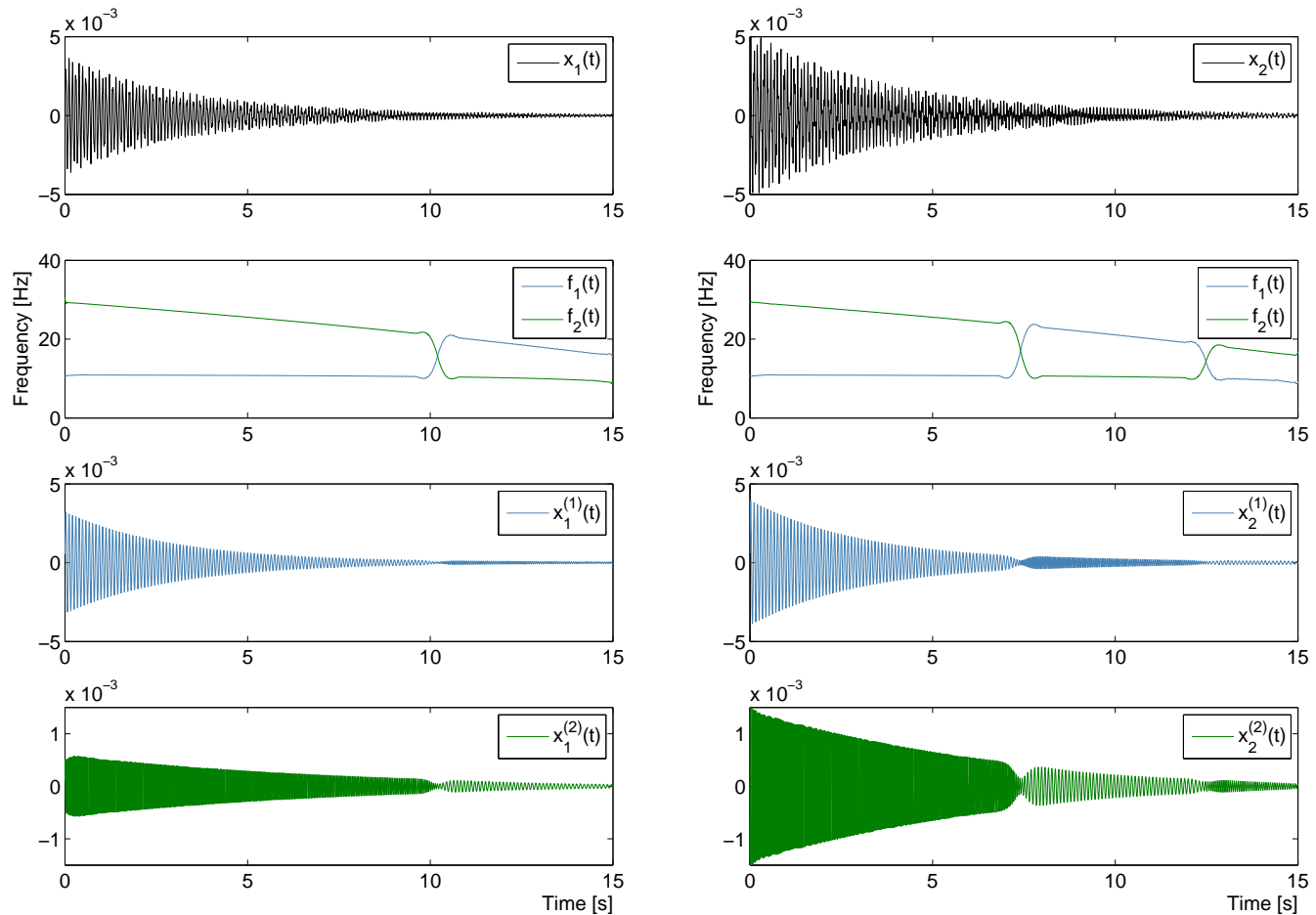

Figure 2: Time responses and extracted instantaneous frequencies and components. First row: time response of each DoF. Second row: identified instantaneous frequencies for each DoF. Third and fourth rows : components corresponding to the identified instantaneous frequencies. 


\section{MODIFIED HILBERT VIBRATION DECOMPOSITION METHOD}

\subsection{Addition of a source separation step to avoid mode switching}

In signal processing, source separation methods are used to recover initial signals, the sources, from a set of recorded signals which are mixtures of the initial sources. A large variety of methods exist trying to separate them as much as possible based on decorrelation, independence or other probabilistic properties. One can cite, among others, the Principal Component Analysis (PCA) (also known as Proper Orthogonal Decomposition (POD) or Karhunen-Loève transform (KLT)), the Smooth Orthogonal Decomposition (SOD), the Independent Component Analysis (ICA) and the Second-Order Blind Source Identification (SOBI). Some of these techniques have been already used in the field of structural dynamics as for example $\mathrm{POD}^{[4]}, \mathrm{SOD}^{[5]}$ and $\mathrm{SOBI}^{[6]}$.

In the present work, the application of a source separation method will help to overcome the previously cited limitations of the HVD method. When several responses recorded on the structure under test are available, the source separation will highlight a particular mode in each identified source. Then, applying the standard steps of the HVD method on the sources rather than directly on the recorded signals will reduce the risk of frequency or amplitude crossing between different modes.

As an example, the identification of the system described in Section 4 was performed again using separated sources (given by the SOD method in the present case) as references for the instantaneous phase identification. As it is observed in Figure 3 , this procedure leads to well decomposed components. Furthermore, while in the previous identification we get four frequency curves (two instantaneous frequencies for each channel), we have this time only two instantaneous frequencies for the whole system, one frequency curve per mode.
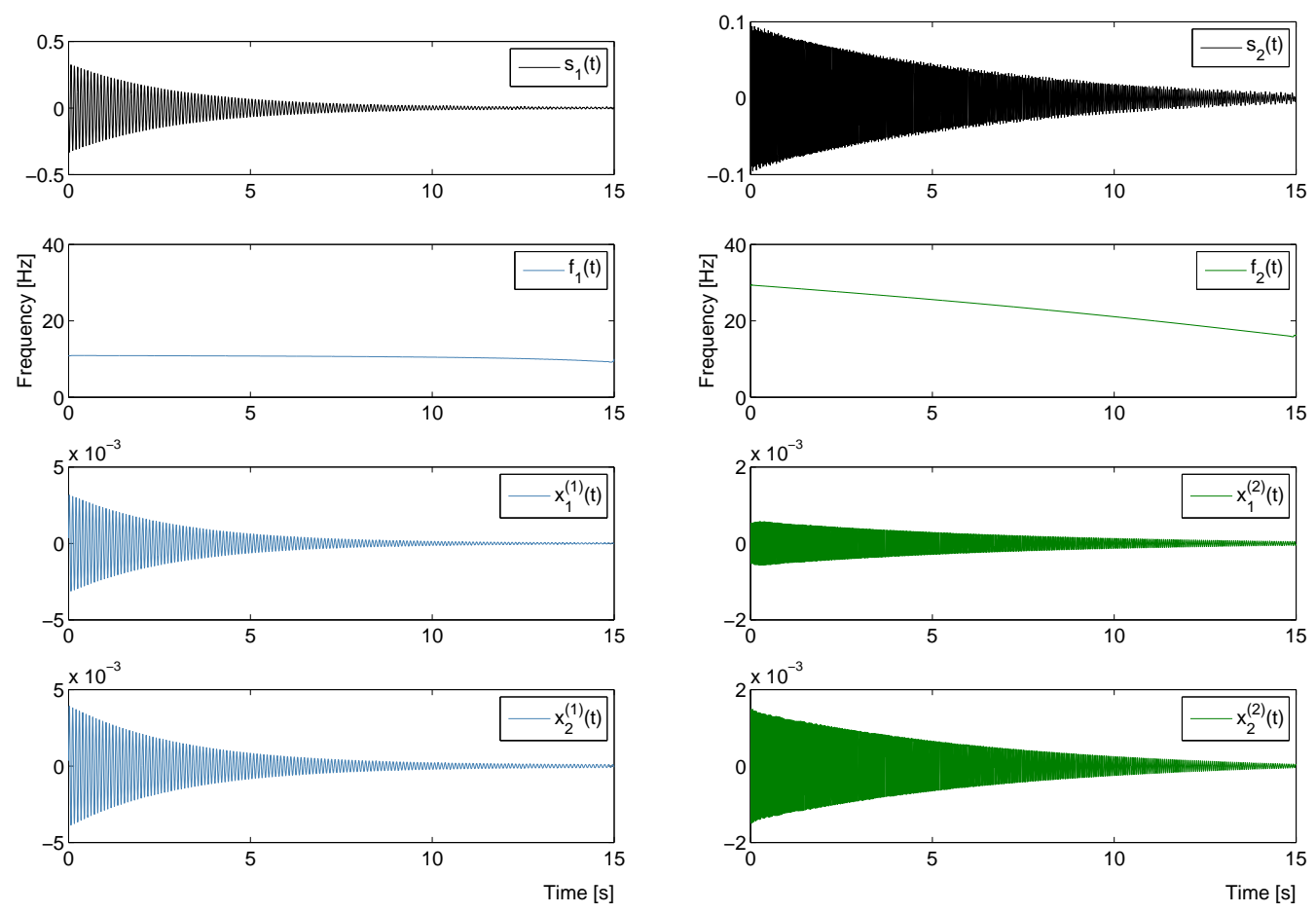

Figure 3: Source separation step introduced in the algorithm. First row: separated sources. Second row: identified instantaneous frequency for each source. Third and fourth rows : components corresponding to the identified instantaneous frequencies for each DoF. 


\subsection{Instantaneous phase/frequency and mode deflection shapes calculation}

Once the source separation method has generated a set of different sources, the Hilbert transform is applied on the first one. The unwrapped phase of the dominant source is computed taking the phase angle of the analytic signal and removing all the $2 \pi$ radians jumps. At this stage the phase curve contains the phase evolution of the dominant mode plus some deviations due to other modes and noise. In the original HVD method, the next steps would be to differentiate the phase to get the instantaneous frequency and low-pass it. In the present approach, we choose to work directly on the phase signal so that the low-pass filter step becomes a trend detection step. For that purpose, several techniques exist such as the Hodrick-Prescott filter ${ }^{[7]}$.

To make a long story short, let us recall that the Hodrick-Prescott filter models the signal (the unwrapped phase $\phi(t)$ in our case) at the summation of a trend $\tau$, oscillatory components $c$ and noise $\nu$ :

$$
\phi(t)=\tau(t)+c(t)+\nu(t)
$$

The goal of the filter is to find the optimal trend $\tau$ that minimizes the following objective function:

$$
\min _{\tau}\left[\sum_{t=1}^{T}\left(\phi_{t}-\tau_{t}\right)^{2}+\lambda \sum_{t=2}^{T-1}\left(\left(\tau_{t+1}-\tau_{t}\right)-\left(\tau_{t}-\tau_{t-1}\right)\right)^{2}\right],
$$

$\lambda$ being a smoothing parameter. This objective function is a trade-off between two parts. The first term of (8) penalizes large variations of the signal around the trend while the second term is a smoothing function penalizing too fast variations of the trend.

Finally a Vold-Kalman filter (VKF $)^{[8]}$ is used instead of synchronous demodulation for its ability of extracting simultaneously a set of mono-components. The Vold-Kalman filter is a technique able to recover from a signal the component(s) $x_{k}(t)$ corresponding to the phase $(\mathrm{s}) \phi_{k}(t)$. The model used for the signal under interest is a summation of components and noise

$$
x(t)=\sum_{k} \underbrace{a_{k}(t) e^{i \phi_{k}(t)}}_{x_{k}(t)}+\nu(t)
$$

in which $a_{k}(t)$ is the complex amplitude of the $k^{t h}$ component $x_{k}(t)$ and $\nu(t)$ is the noise in the signal.

The way to recover the components $x_{k}(t)$ is based on the minimization of two equations. First the data equation

$$
x(t)-\sum_{k} a_{k}(t) e^{i \phi_{k}(t)}=\delta(t)
$$

in which the error $\delta(t)$ should be minimal. The second one is the structural equation ensuring the smoothness of the solution using the difference operator $\nabla^{p+1}$ applied on $a_{k}(t)$

$$
\nabla^{p+1} a_{k}(t)=\varepsilon_{k}(t)
$$

where $\varepsilon_{k}(t)$ should also be minimized.

All the complex amplitudes $a_{k}(t)$ are computed in a least square sense considering these two set of equations. Now making a parallelism between equation (9) and modal expansion, we can equate the complex amplitude of the component $k$ from (9) to the unscaled time-varying component in the $k^{\text {th }}$ mode of vibration.

$$
\begin{array}{cccc}
\text { Vold-Kalman filter: } & \boldsymbol{x}(t)=\sum_{k} & \boldsymbol{a}_{\boldsymbol{k}}(t) & e^{i \phi_{k}(t)} \\
& & \uparrow & \uparrow \\
\text { Modal expansion: } & \boldsymbol{x}(t)=\sum_{k} & \boldsymbol{V}_{\boldsymbol{k}}(t) & \eta_{k}(t)
\end{array}
$$

The full algorithm of the method is illustrated in a block diagram in Figure 4.

\section{NUMERICAL APPLICATION}

The numerical application considered in this study consists of a beam on which a moving mass is traveling. The beam is two meters-long and is simply supported at both ends; two random external force excitations are applied in the vertical and lateral 


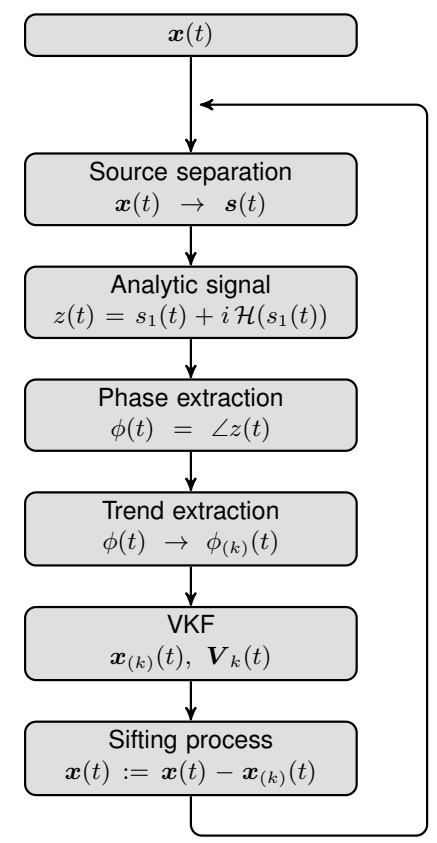

Figure 4: Flow chart of the method.

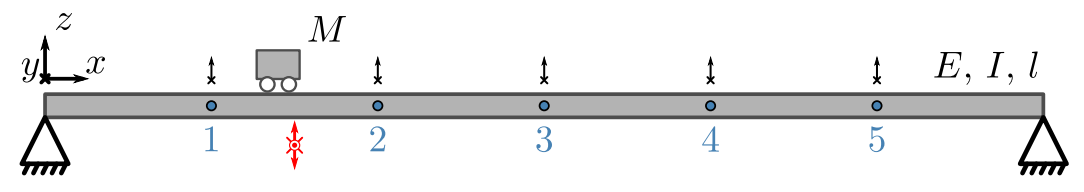

Figure 5: Beam travelled by the moving mass

directions at a quarter of the total length of the beam. Five equally spaced measurement points are selected to record the time response of the beam in the vertical and lateral directions. A mass of $3 \mathrm{~kg}$ travels the whole beam in 60 seconds during which the external forces are applied. The response of the system is computed using the LMS-Samcef Mecano ${ }^{[9]}$ software in which a slider element is used to make the connection between the beam and the lumped mass. The measured time responses are sampled at a rate of $1000 \mathrm{~Hz}$ and to better simulate a real measurement process, a normally distributed noise is added on each signal with a signal-to-noise ratio of $1 \%$. The simulated system and the measurement set-up are illustrated in Figure 5 . The properties of the system are the following:

- Beam length: $l=2 \mathrm{~m}$

- Beam cross section $80 \mathrm{~mm}$ (width) $\times 20 \mathrm{~mm}$ (height)

- Density : $\rho=2700 \mathrm{~kg} / \mathrm{m}^{3}$

- Young's modulus : $E=70000 \mathrm{MPa}$

- Poisson's ration: 0.33

- Moving mass: $3 \mathrm{~kg}$

- Pinned connections: $x(0)=y(0)=z(0)=x(l)=y(l)=z(l)=0$ 


\begin{tabular}{ccc}
\hline Mode Nbr. & Frequency [Hz] & Mode shape \\
\hline \hline Mode 1 & 11.54 & First vertical bending \\
Mode 2 & 46.05 & First lateral bending \\
Mode 3 & 46.15 & Second vertical bending \\
Mode 4 & 103.74 & Third vertical bending \\
Mode 5 & 182.72 & Second lateral bending \\
\hline
\end{tabular}

TABLE 1: Modal parameters of the simply supported beam only (LTI system)

The eigenfrequencies of the beam subsystem are listed in Table 1 with their corresponding mode-shapes.

Using cross correlation between time signals, it can be verified that the responses in the lateral and vertical directions are completely separated, so that the two sets of measurements can be treated separately. In Figure 6 wavelet spectra of the response of the first node in both lateral (6(a)) and vertical (6(b)) directions are shown. In this figure, the white dashed lines correspond to the natural frequencies of the beam subsystem (when the mass is located on one support, before beginning its motion). It can be observed that, for a given mode, the natural frequency decreases as the mass is moving and comes back to its initial value every time the mass passes upon a vibration node. This is due to the fact that the additional inertia force induced by the moving mass does not produce any work on that mode at this time. Conversely, when the mass is located at an anti-node of vibration for a particular mode, the inertia effect is maximum and produces the maximum decay in frequency for that mode.

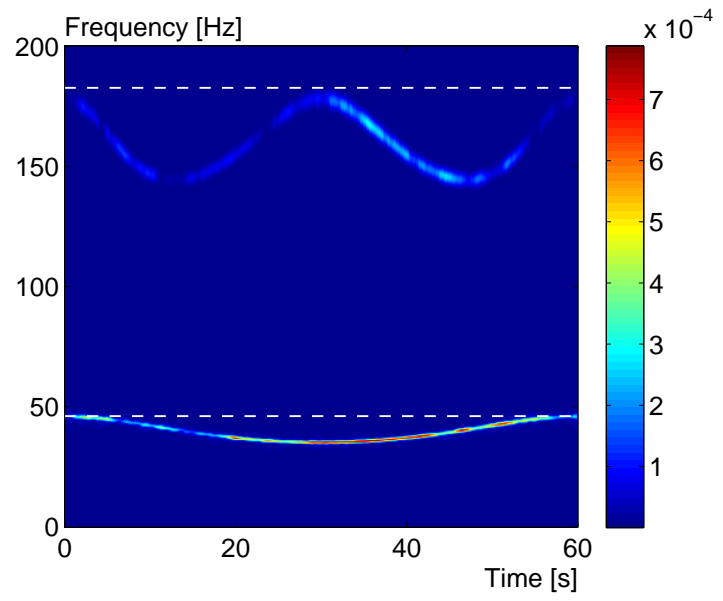

(a) Node 1 - Lateral direction

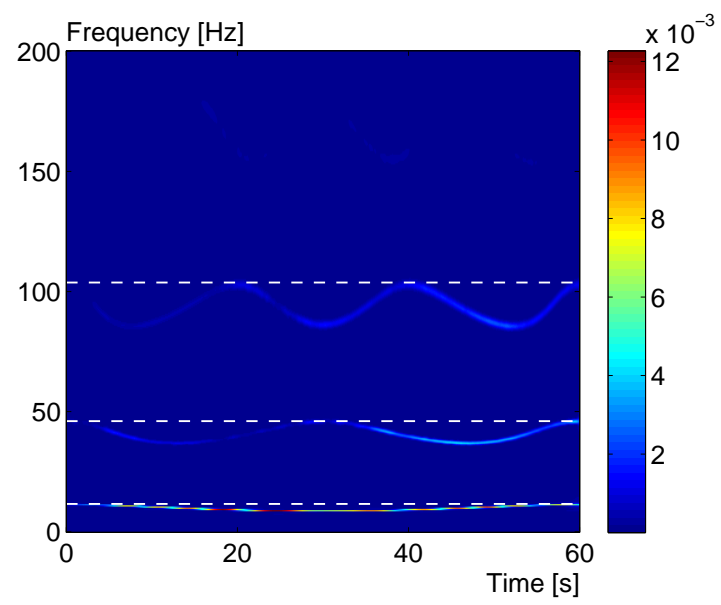

(b) Node 1 - Vertical direction

Figure 6: Wavelet spectra of two degrees of freedom. The time varying behaviour of the structure is clearly visible.

\subsection{Identification of instantaneous frequencies}

The algorithm proposed in Figure 4 is now applied on each set of recorded signals. Based on the time-frequency plots $6(\mathrm{a})$ and $6(b)$ it is chosen to extract two modes from the set of lateral measurements and three modes from the vertical ones.

The evolution of the five identified instantaneous frequencies are shown using white dashed curves in Figures 7 (a) and 7 (b) for the lateral and vertical modes respectively. Wavelet spectra of node 1 are used in the background of the plots and it can be seen that the identified instantaneous frequencies match very well the highest amplitude in the spectra. 


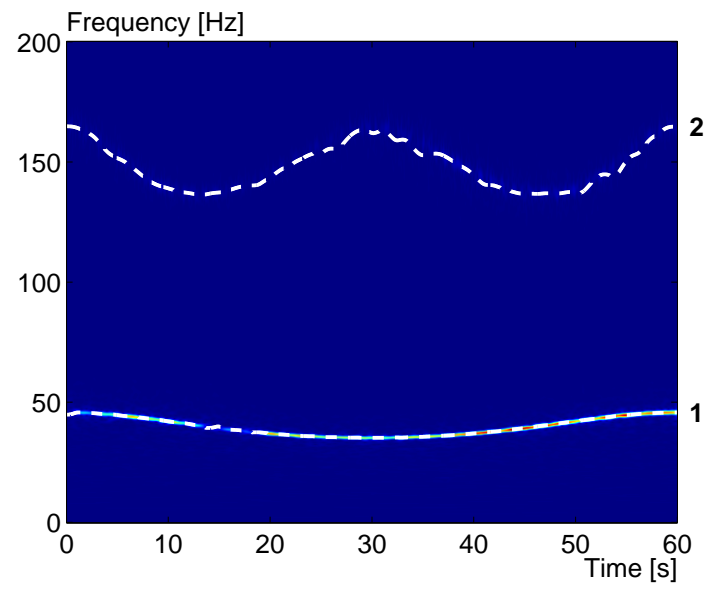

(a) Instantaneous frequencies of lateral bending modes.

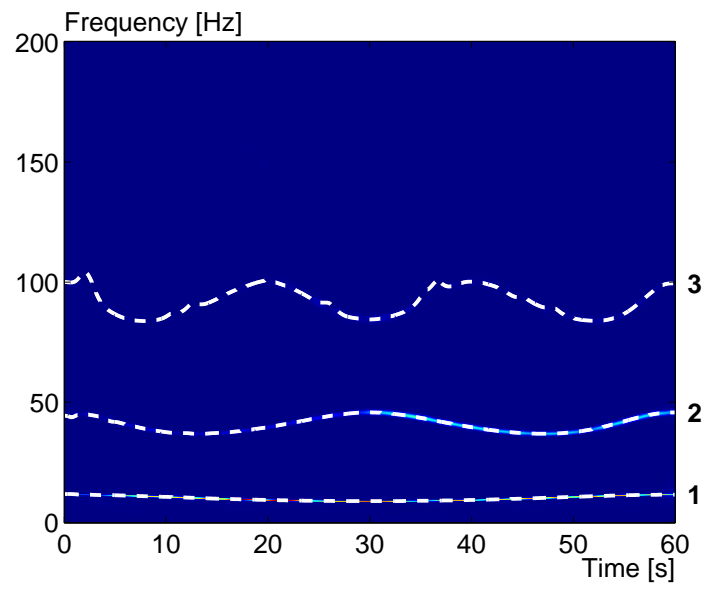

(b) Instantaneous frequencies of vertical bending modes.

Figure 7: Identified instantaneous frequencies. (Numbers on the right of each time-frequency plot indicates the sequence of the modes extraction.)

\subsection{Component extraction and calculation of mode deflection shapes}

Once that the instantaneous eigenfrequencies are well identified, the next step is to extract the intrinsic components corresponding to each frequency in all the channels. This is done by the use of the Vold-Kalman filter and results in a set of mono-components and complex amplitudes. Referring to (9), these complex amplitudes are used as unscaled instantaneous mode-shapes.

For seek of illustration, the three first vertical bending modes obtained at three different times are given in Figure 8 along with the mode-shapes of the beam subsystem. Note that the lateral bending modes show the same behavior but are not reported here. In the first row of Figure 8, the considered time is 15 seconds. At this time, the mass is located at a quarter of the length of the beam, which corresponds to an anti-node of vibration for the second mode. As seen in Figure 8 (b), the second mode is highly perturbed by the moving mass.

In Figures 8(f) and 8(h), the deformed shapes corresponding to modes 3 and 2 at 20 and 30 seconds respectively are shown. In both configurations, the mass is located at a node of vibration for the considered mode and the instantaneous mode-shape is similar to the corresponding mode-shape of the beam subsystem.

Finally, Figures $8(\mathrm{a}), 8(\mathrm{~d})$ and $8(\mathrm{~g})$ reveal that the first bending mode is not very sensitive to the presence of the moving mass.

From the set of identified instantaneous mode-shapes, it is possible to perform a correlation with the mode-shapes of the beam subsystem using the classical modal assurance criterion (MAC). As the instantaneous modes are identified at each time step, the time dimension has to be taken into account. So for each time step, the MAC matrix between the identified mode-shapes and the mode-shapes from the finite element analysis (FEA) of the beam subsystem is reshaped in a column vector. In Figure 9, the instantaneous MAC values are shown between identified bending modes in the lateral (9(a)) and vertical (9(b)) directions respectively.

The previously observed perturbations of the mode-shapes due to the presence of the moving mass at vibration nodes or antinodes are also visible in Figures 9(a) and 9(b). As for the identified instantaneous frequencies, the time-varying MAC values drop periodically when the mass is passing on an antinode of vibration and come back close to unity when the mass is passing on a node of vibration. 


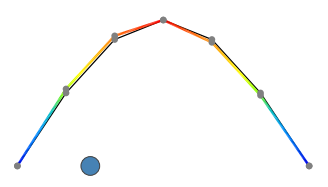

(a) Mode $1-t=15 \mathrm{~s}$.

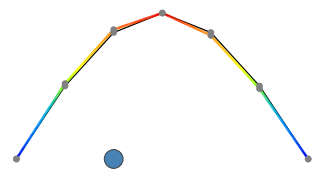

(d) Mode $1-t=20 \mathrm{~s}$.

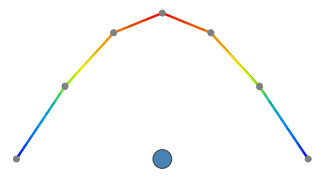

(g) Mode $1-t=30 \mathrm{~s}$.

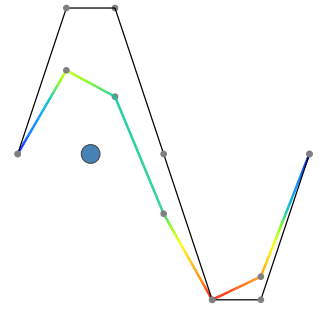

(b) Mode $2-t=15 \mathrm{~s}$.

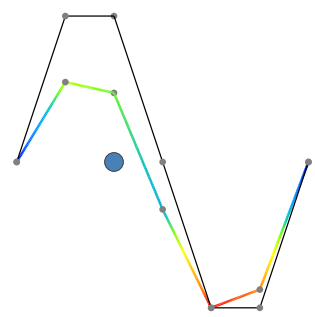

(e) Mode $2-t=20 \mathrm{~s}$.

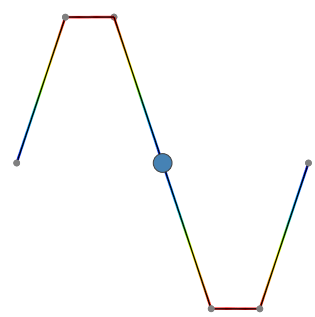

(h) Mode $2-t=30 \mathrm{~s}$.

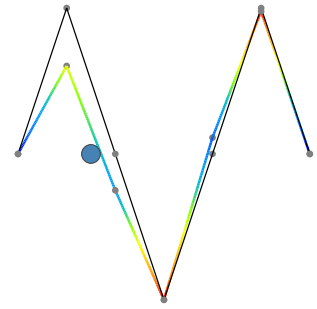

(c) Mode $3-t=15 \mathrm{~s}$.

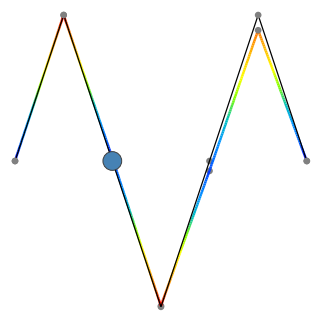

(f) Mode $3-t=20 \mathrm{~s}$.

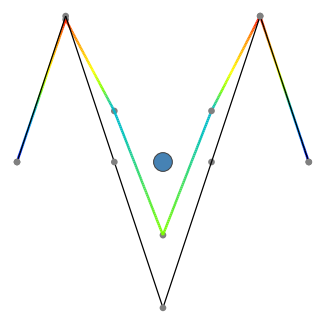

(i) Mode $3-t=30 \mathrm{~s}$.

Figure 8: Plots of the vertical bending mode-shapes at three times: 15, 20 and 30 seconds. The mode-shapes of the beam subsystem are plotted in black and the instantaneous mode-shapes of the time-varying system in color. The blue dot shows the location of the mass for the three considered times. 


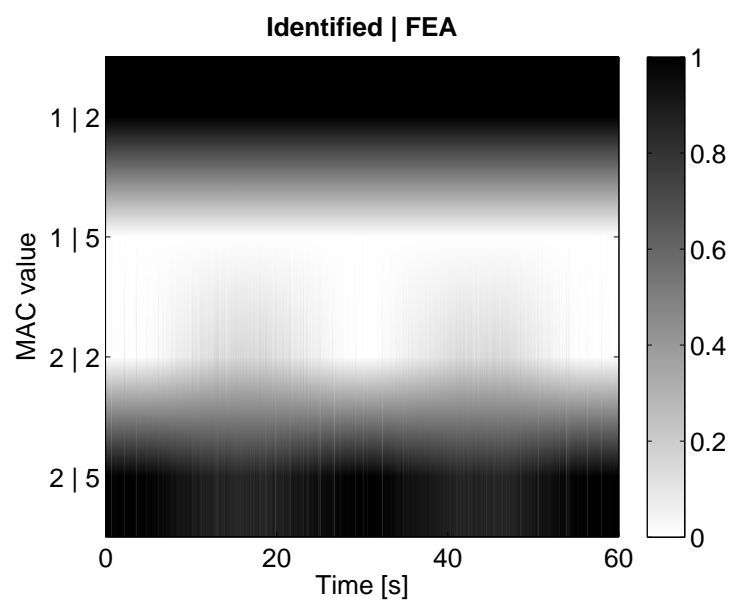

(a) Lateral bending modes.

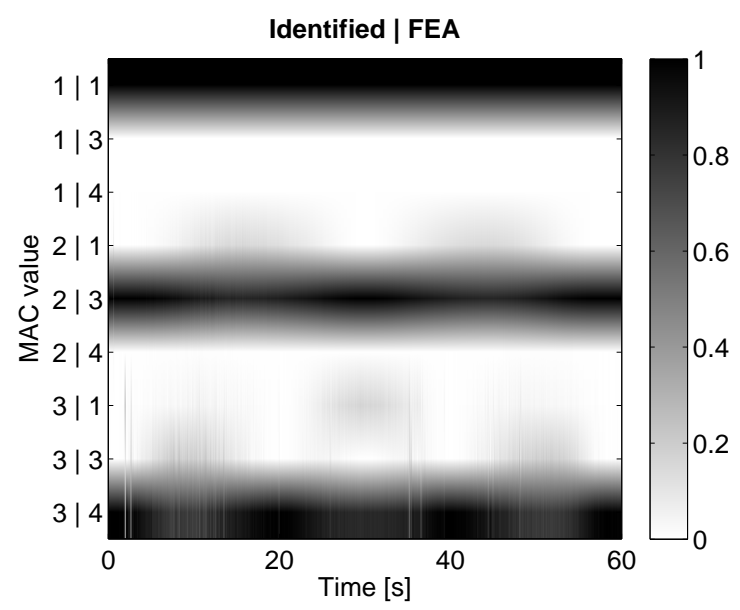

(b) Vertical bending modes.

Figure 9: Modified MAC matrix to take the time variability into account.

\section{CONCLUSION}

In this paper, two well known techniques (the HHT and the HVD methods) used for non-stationary signal decomposition were considered and their limitations in the case of crossing frequencies or amplitudes of intrinsic components were highlighted. As these methods work on single signals, it was shown that, in the case of a multiple degree-of-freedom system, applying them separately on each channel can lead to non-unique frequency curves and consequently to non-corresponding demodulated components on all the channels. To alleviate this problem, a source separation technique was introduced into the algorithm of the original HVD method. It was shown that both limitations were removed as a single frequency curve was calculated for each mode. Thanks to the better estimation of the instantaneous frequencies, mono-components and instantaneous mode-shapes were calculated.

\section{REFERENCES}

[1] Huang, N. E., Shen, Z., Long, S. R., Wu, M. C., Shih, H. H., Zheng, Q., Yen, N.-C., Tung, C. C. and Liu, H. H., The empirical mode decomposition and the Hilbert spectrum for nonlinear and non-stationary time series analysis, Proceedings of the Royal Society A: Mathematical, Physical and Engineering Sciences, Vol. 454, No. 1971, pp. 903-995, Mar. 1998.

[2] Feldman, M., Hilbert Transform Applications in Mechanical Vibration, John Wiley \& Sons, Ltd, Chichester, UK, Mar. 2011.

[3] Bedrosian, E., A product theorem for Hilbert transforms, Proceedings of the IEEE, Vol. 51, No. 5, pp. 868 - 869, 1963.

[4] Kerschen, G. and Golinval, J., Physical Interpretation of the Proper Orthogonal Modes Using the Singular Value Decomposition, Journal of Sound and Vibration, Vol. 249, No. 5, pp. 849-865, Jan. 2002.

[5] Chelidze, D. and Zhou, W., Smooth orthogonal decomposition-based vibration mode identification, Journal of Sound and Vibration, Vol. 292, No. 3-5, pp. 461-473, May 2006.

[6] Poncelet, F., Kerschen, G., Golinval, J.-C. and Verhelst, D., Output-only modal analysis using blind source separation techniques, Mechanical Systems and Signal Processing, Vol. 21, No. 6, pp. 2335-2358, Aug. 2007.

[7] Alexandrov, T., Bianconcini, S., Dagum, E. B., Maass, P. and McElroy, T., A review of some modern approaches to the problem of trend extraction, Statistics, 2008.

[8] Feldbauer, C. and Höldrich, R., Realization of a Vold-Kalman Tracking Filter - A Least Squares Problem, Proceedings of the COST G-6 Conference on Digital Audio Effects (DAFX-00), No. 8, pp. 8-11, Verona, Italy, 2000.

[9] SAMCEF Mecano, http://www.Imsintl.com/samcef-non-linear-motion. 